ОПРЕДЕЛЕНИЕ ИНДУКТИВНОСТИ

ЭКИПАЖНОГО ЭЛЕКТРОМАГНИТА

МАГНИТОЛЕВИТАЦИННОЙ

ТРАНСПРОРТНОЙ СИСТЕМЫ

Г. Е. Середа, В. М. Стрепетов

Петербургский государственный университет путей сообщения Императора Александра I

(Санкт-Петербург, Россия)

DEFINITION OF INDUCTANCE VEHICLE ELECTROMAGNET MAGNITOLEVITATION TRANSPRORT SYSTEM

G. E. Sereda, V. M. Strepetov Emperor Alexander I St.

Petersburg State Transport University

(St. Petersburg, Russia)

Аналитическое исследование установившихся и переходных режимов работы различных магнитолевитационных транспортных системах, в том числе и относительно мало исследованной в комбинированной системе левитации и тяги на переменном токе предполагает знание параметров этих систем и в частности индуктивности экипажных электромагнитов. При этом точность определения коэффициента самоиндукции во многом определяет достоверность результатов, получаемых при исследовании электромеханических систем, в особенности, если эти исследования носят поисковый характер.

Расчёт коэффициента самоиндукции различных проводящих систем, как правило, представляет собой технически сложную процедуру, связанную с громоздкими вычислениями даже для самых простых форм электромагнитов.Большое количество справочников по вычислению указанных величин (см., например, библиографию в [1-2]) содержат, в основном, наборы приближённых формул, точность которых и зоны их применимости далеко не всегда указываются. 
Настоящая статья посвящена определению величины коэффициента самоиндукции $L_{T}$ для проводников определённой конфигурации, у которых величина «высоты» электромагнита пренебрежимо мала по сравнению с другими геометрическими размерами катушки. Такие электромагниты будем называть «бесконечно» тонкими (плоскими) источниками магнитного поля.

Общая формула для определения индуктивности плоского источника магнитного поля $L_{T}$ задаётся следующим соотношением [2]:

$$
L_{T}=\frac{\mu_{0} W^{2}}{4 \pi I^{2}} \int_{S} d \boldsymbol{\rho} \int_{S^{\prime}} d \boldsymbol{\rho}^{\prime}\left(\boldsymbol{i}, \boldsymbol{i}^{\prime}\right) /\left|\boldsymbol{\rho}-\boldsymbol{\rho}^{\prime}\right|
$$

где $\quad d \boldsymbol{\rho}=d x d y, \quad d \boldsymbol{\rho}^{\prime}=d x^{\prime} d y^{\prime}, \quad\left|\boldsymbol{\rho}-\boldsymbol{\rho}^{\prime}\right|^{2}=\left(x-x^{\prime}\right)^{2}+\left(y-y^{\prime}\right)^{2}$ $(x, y)$ и $\left(x^{\prime}, y^{\prime}\right)$ - подходящие декартовы координаты, $\boldsymbol{i}$ и $\boldsymbol{i}^{\prime}-$ линейные плотности тока, $S \equiv S^{\prime}$ - токонесущая поверхность катушки, $W$ - число витков, $\mu_{0}=4 \pi \cdot 10^{-7} \Gamma \mathrm{H} / \mathrm{M}-$ магнитная постоянная. Дальнейшие рассмотрения будут проводиться для одновитковой катушки $(W=1)$. В качестве плоского источника магнитного поля рассмотрим электромагнит прямоугольной формы, схематичное изображение которого представлено на рис.1.

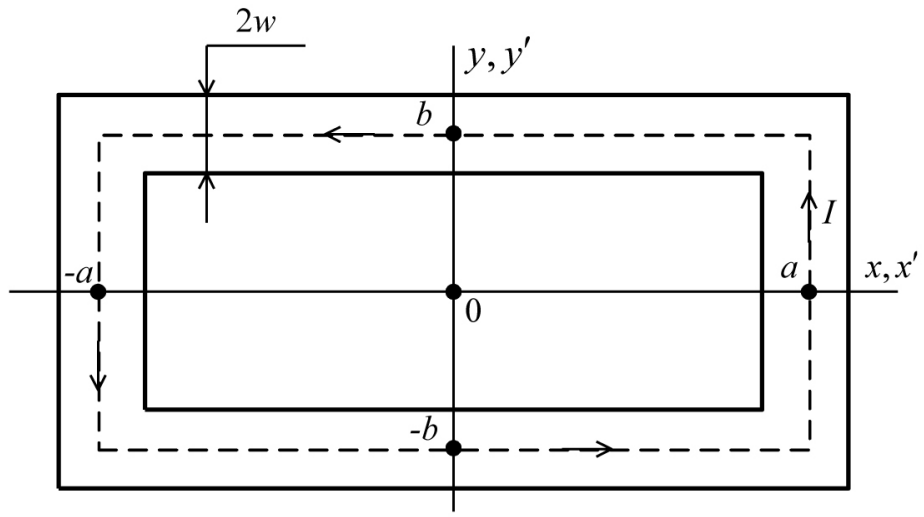

Рис.1. Схематическое изображение плоского источника магнитного поля прямоугольной формы 
Смысл обозначений, указанных на рис. 1 понятен из его содержания. Отметим, что на геометрические параметры данного плоского электромагнита накладывается естественное ограничение: $0<w \leq \min (a, b)$.

Для рассматриваемой конфигурации источника магнитного поля модуль линейной плотности тока определяется равенством $i=i^{\prime}=I / 2 w$. Переходя в (1) к безразмерным координатам и совершая там же первичное двукратное интегрирование, можно получить следующее выражение для коэффициента самоиндукции плоской катушки прямоугольной формы $L_{\mathrm{T}}$ :

Переходя в (1) к безразмерным координатам и совершая там же первичное двукратное интегрирование, можно получить следующее окончательное выражение для коэффициента самоиндукции плоской катушки прямоугольной формы $L_{\mathrm{T}}$ :

$$
\frac{L(v)}{v}=\ln \frac{1+r}{\varepsilon}-\frac{1}{\varepsilon} \ln (\varepsilon+r)-\frac{(2+r)^{2}-2}{3(1+r)}-\frac{1}{3 \sqrt{2} \varepsilon^{2}} \sum_{k= \pm 1}(1+k \varepsilon)^{3} \ln \frac{1+\sqrt{2} r-k \varepsilon}{(1+\sqrt{2})(1+k \varepsilon)}
$$

где $r^{2}=1+\varepsilon^{2}, \varepsilon=\delta / \nu,(0<\varepsilon \leq 1)$

В качестве нормирующего множителя при переходе к безразмерным координатам в (2) выбрана величина $2 p-$ полупериметр катушки по средней линии.

Расчёт величины $L_{T}$ целесообразно проводить в безразмерном виде. В качестве базовой выберем величину индуктивности плоской катушки $L_{0}$, представляющей собой квадрат с «проколотым» центром. После соответствующих вычислений при $\alpha=\beta=\delta$ можно получить приближенную формулу

$$
L_{0}=\frac{\mu_{0} P}{\pi} \cdot \frac{2}{3} \cdot \frac{1+\ln (1+\sqrt{2})}{1+\sqrt{2}} \approx 2.078 \cdot 10^{-7} P,
$$

$P=4 p-$ периметр квадратной катушки по средней линии.

Результаты численного анализа, показывающего зависимость относительной индуктивности $L_{T} / L_{0}$ от 
параметра е при различных значениях «вытянутости» катушки представлены на рис.2.

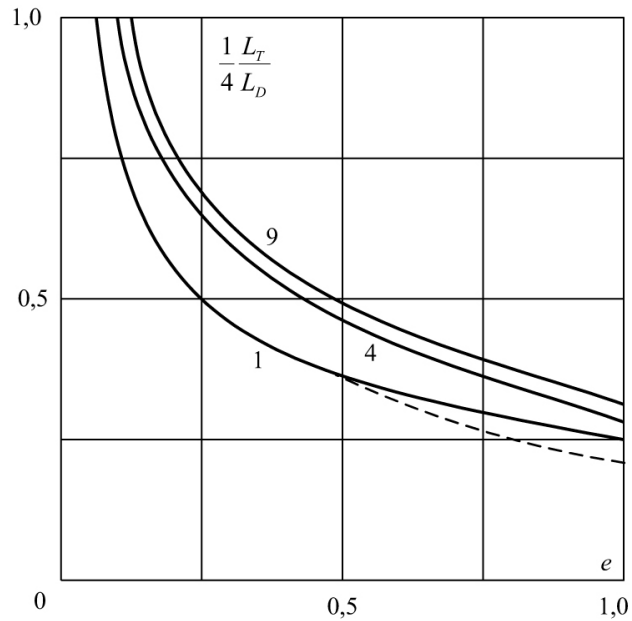

Рис.2. Зависимость относительного коэффициента самоиндукции плоской катушки прямоугольной формы $L_{T} / L_{0}$

(числа у кривых соответствуют значениям «вытянутости» катушки). Параметры $e$ и $\zeta$ соответственно равны: $e=w / \min (a, b), \varsigma=\max (a, b) / \min (a, b)$. Пунктирная линия отвечает приближённой формуле $\left.L\right|_{\delta<<\min (\alpha, \beta)} / L_{0}$ при $\varsigma=1$. В расчётах периметр катушки по средней линии $P=4 p$ принимается постоянным, $L_{0}$ определено в (14).

\section{Краткие выводы:}

1. Получено точное аналитическое выражение для величины коэффициента самоиндукции «тонкого» источника магнитного поля прямоугольной формы в виде алгебраической суммы элементарных функций.

2. Выведена приближённая формула для вычисления коэффициента самоиндукции, погрешность которой не 
превышает 14\% в области изменений всех геометрических параметров электромагнита.

\section{Библиографический список}

1. Калантаров П.Л., Цейтлин Л.А. Расчет индуктивностей.- Л.: Энергоиздат, 1986.- 488 с.

2.Матвеев А.Н. Электродинамика - М.: Высш. школа, 1980.- $383 \mathrm{c}$.

3. Немцов М.В., Шамаев Ю.М. Справочник по расчету параметров катушек индуктивности. Москва, издательство Энергоиздат, 1981.- $136 \mathrm{c.}$

\section{Сведения об авторах:}

Середа Геннадий Евгеньевич E-mail: gennady.sereda@mail.ru

Стрепетов Владимир Михайлович

E-mail: strepetov.vm@mail.ru

\section{Information about authors:}

Gennady E. Sereda

E-mail: gennady.sereda@mail.ru

Vladimir M. Strepetov

E-mail: strepetov.vm@mail.ru 